\title{
The effect of Bongardia Chrysogonum on prostate tissue in a rat model of STZ-induced diabetes
}

\author{
Recep Dokuyucu ${ }^{1 *}$, Kerem Han Gozukara², Oguzhan Ozcan ${ }^{3}$, Nebihat Kaplan Sefil ${ }^{4}$, Ahmet Nacar ${ }^{5}$, \\ Ahmet Dokuyucu ${ }^{6}$ and Mehmet Inci ${ }^{2}$
}

\begin{abstract}
Background: Bongardia chrysogonum is widely used in Turkey for treating urinary tract infections and prostate hypertrophy, and it also has potent hypoglycemic effects and aids glucose homeostasis. Because of the inflammatory conditions in diabetes mellitus (DM), the prostate tissue of men with diabetes is particularly susceptible to developing hypoplasia, and DM produces characteristic pathological changes in prostate tissue. Here, we examined the effects of B. chrysogonum on the prostate tissue of rats with streptozotocin (STZ)-induced diabetes.

Results: The glucose levels were statistically significantly higher in the diabetic rats than in healthy controls $(P<0.001)$. Further, they were significantly lower in the healthy and diabetic rats administered $B$. chrysogonum than in the untreated diabetic rats $(P<0.001$ and 0.05 , respectively). The total cholesterol levels were significantly lower in the healthy rats administered $B$. chrysogonum than the healthy controls $(P<0.05)$ and diabetic rats $(P<0.01)$. They were also significantly lower in the diabetic rats administered B. chrysogonum than those that were left untreated $(P<0.05)$. The testosterone levels were significantly lower in the untreated diabetic rats than in the controls (untreated ones and those administered B. chrysogonum) and diabetic rats administered the herb ( $P<0.001,0.05$ and 0.01 , respectively). The oxidative stress index was significantly higher in the untreated diabetic rats than the healthy controls $(P<0.05)$. It was also significantly lower in the healthy and diabetic groups treated with B. chrysogonum than the untreated diabetic rats $(P<0.05)$. Histological examination showed no changes in the prostate tissue of the non-diabetic rats. In the diabetic group, the glandular lumens were filled with cellular debris and leucocytic infiltrate, and the glandular epithelium was degenerated and thickened. In the diabetic group treated with B. chrysogonum, the epithelium was better preserved and less debris was seen in the glandular lumen.
\end{abstract}

Conclusion: To our knowledge, this is the first study to histologically prove the effects of $B$. chrysogonum on prostate tissue in diabetes. Our findings may be useful in developing B. chrysogonum into a therapeutic agent against diabetes and benign prostate hyperplasia.

Keywords: Diabetes mellitus, Prostate, Bongardia chrysogonum, Testosterone, Oxidative stress, Inflammation

\section{Background}

Benign prostatic hyperplasia $(\mathrm{BPH})$ is characterized by narrowing of the urethral opening as a result of the growth of prostate tissue around the urethra (COMMITTEE 2003). Histological BPH is observed

\footnotetext{
*Correspondence: drecepfatih@gmail.com

${ }^{1}$ Department of Medical Physiology, School of Medicine, Mustafa Kemal University, 31100 Antakya, Hatay, Turkey

Full list of author information is available at the end of the article
}

in $8 \%$ of men between 31 and 40 years of age, the incidence increases markedly with age, and reaches about $90 \%$ in the 9th decade of life (Rosen et al. 2003, 2005), and this condition greatly hampers quality of life. Although most literature implicates non-controllable factors like age, hormonal status, and genetics in $\mathrm{BPH}$, newer publications link it to metabolic factors such as high blood glucose and blood lipid levels, and the field is rapidly advancing (Parsons 2007). Thus, new targets for 
prevention and treatment of $\mathrm{BPH}$ may be revealed in the near future.

As with $\mathrm{BPH}$, the incidence of diabetes mellitus (DM) increases with age, and developed communities need to allocate a large share of healthcare expenses toward these diseases. Between 3.5 and 4.2 million men in the United States have both BPH and DM. When treating either of these diseases, however, the possible presence of other pathologies must be kept in mind, as the population most commonly affected by them is the elderly (Klein et al. 2005).

Bongardia chrysogonum is a tuberous plant used in the treatment of certain diseases in south-east Anatolia. Its use particularly in the treatment of urinary tract infections, prostate hypertrophy, hypercholesterolemia, epilepsy, and cancer has increased its national demand, and it is marketed throughout Turkey (Arslan et al. 2005). $B$. chrysogonum also reportedly has potent hypoglycemic effects and is useful for glucose homeostasis, in addition to multiple anti-inflammatory effects exerted via saponins (Arslan et al. 2005). Saponins are fat- and watersoluble glycosides that combine with glycan or aglycan components of B. chrysogonum. They inhibit the emulsification of fat molecules and bile acids via reducing their surface tension and detergent effects in the gastrointestinal tract. Another well-defined anti-hypercholesterolemic mechanism of saponins is the ability to change the quantity and dimension of cholesterol micelles, which reduces their absorption by intestinal mucosa cells (Rahman et al. 2000; Rao and Kendall 1986; Sidhu and Oakenfull 1986; Whitehead et al. 1981), with consequent effects on metabolism.

In the present study, we examined the effects of $B$. chrysogonum on prostate tissue in a rat model with streptozotocin (STZ)-induced diabetes in terms of the histological and biochemical aspects.

\section{Methods}

The ethics committee of Mustafa Kemal University approved the animal use protocol for this study, which is in compliance with the Declaration of Helsinki (ID: 40595970/126). Male Wistar albino rats were used (250$300 \mathrm{~g}$ ) in this study. They were housed in a $12 \mathrm{~h}$ light and dark environment at room temperature. During the experiment, the rats were provided normal mouse pellets and water. Throughout the experiment, the animal conditions were maintained according to international ethical guidelines for laboratory animals.

\section{Animal protocol}

Forty adult male Wistar albino rats were divided into four groups [control (C), diabetes group (D), B. chrysogonum group (B), and diabetes $+B$. chrysogonum group
(DB)] of 10 rats each. During the test period, the rats in all groups were given normal mouse pellets and water. At the beginning of the experiment, the blood sugar levels in all animals were measured using a glucometer. The rats in groups D and DB were administered $60 \mathrm{mg} / \mathrm{kg} \mathrm{STZ} \mathrm{intra-}$ venously (prepared by dissolving STZ in citrate buffer). After $72 \mathrm{~h}$, the urine of rats from groups D and DB was examined for glucose levels using strip analyzers. Rats with blood glucose concentrations $\geq 300 \mathrm{mg} / \mathrm{dL}$ (and for the urine samples, the color of the strip changed from yellow to green) were considered diabetic (Gulturk et al. 2010).

\section{Preparation of $B$. chrysogonum infusion}

Bongardia chrysogonum obtained from a local herbalist was crushed, and an infusion was prepared by passing $100 \mathrm{~mL}$ of boiled water through $3 \mathrm{~g}$ of this material. A fresh infusion was prepared each week before the test phase (Arslan et al. 2005).

\section{Administration of $B$. chrysogonum}

The rats in groups B and DB were administered $0.2 \mathrm{~mL}$ of the infusion obtained as mentioned above by oral gavage once every day for 5 weeks (Arslan et al. 2005). The rats in groups $\mathrm{C}$ and $\mathrm{D}$ were similarly administered $0.2 \mathrm{~mL}$ of water. At the end of the 5-week experiment, all rats were anesthetized using ketamine $(90 \mathrm{mg} / \mathrm{kg})$ and xylazine (3 mg/kg).

\section{Hematological evaluation}

Cardiac blood samples were collected, and the serum levels of glucose, total cholesterol (TC), high-density lipoprotein (HDL), low-density lipoprotein (LDL), and triglycerides (TG) were measured using spectrophotometry.

\section{Measurement of total antioxidant status}

Serum total antioxidant status (TAS) levels were measured using the new automated method developed by Erel (2004). This assay has excellent precision $(<3 \%)$. In this method, the most powerful hydroxyl radicals are generated first. Reagent 1 containing ferrous ion solution is mixed with reagent 2 , which includes hydrogen peroxide, $\mathrm{n}$ the assay. The brown-colored dianisidine radical cation is a strong radical produced by hydroxyl radicals. Nonpotent radical reactions initiated by the hydroxyl radicals produced because of antioxidative effect of working examples were also measured. The results are expressed in mmol Trolox equivalents/L.

\section{Measurement of total oxidant status}

Serum total oxidative status (TOS) levels were also measured using the method of Erel (2005). In this method, oxidants present in the samples oxidize the ferrous 
ion-o-dianisidine complex to ferric ions. The oxidation reaction is enhanced by abundant glycerol molecules in the reaction medium. The ferric ions form a colored complex with xylenol orange in an acidic medium. The color intensity, which can be measured spectrophotometrically, is related to the total amount of oxidant molecules in the sample. The assay was calibrated using hydrogen peroxide. The results are expressed in terms of micromoles of hydrogen peroxide equivalents per liter $(\mu \mathrm{mol}$ $\mathrm{H}_{2} \mathrm{O}_{2}$ equivalents/L).

\section{Oxidative stress index}

The percentage of the TOS to TAS ratio was used as the oxidative stress index $(\mathrm{OSI})$ value $[\mathrm{OSI}=\mathrm{TOS}(\mu \mathrm{mol}$ $\mathrm{H}_{2} \mathrm{O}_{2}$ equivalents/L)/TAS (mmol Trolox equivalents/L)] (Dokuyucu et al. 2014).

\section{Histopathological assessment}

Renal and prostate samples were fixed in $10 \%$ neutral buffered formalin for light microscopic examination. After the tissues were dehydrated in a graded alcohol series and embedded in paraffin, 5 - $\mu$ m-thick transverse sections were prepared from the tissue blocks and stained with hematoxylin-eosin and reticulin for histologic evaluation. Sections were examined for characteristic changes and photographed using an Olympus DP20 camera attached to an Olympus CX41 photomicroscope (American Diabetes Association 1998; McVary 2006a, b).

\section{Statistical analysis}

Statistical analysis was performed using GraphPad software, version 5.0 (GraphPad Inc., La Jolla, CA, USA). Normally distributed continuous variables were tested using one-way ANOVA and the post hoc Student's $t$ test. P values less than 0.05 were considered statistically significant.

\section{Results}

\section{Glucose, TC, TG, HDL, LDL, and testosterone levels}

The glucose levels were significantly higher in group $\mathrm{D}$ than in group $\mathrm{C}(\mathrm{P}<0.001)$. Further, they were significantly lower in groups $\mathrm{B}$ and $\mathrm{DB}$ than in group $\mathrm{D}$ $(\mathrm{P}<0.001$ and 0.05 , respectively) (Table 1$)$. No significant differences were found in the TC, TG, LDL, or HDL levels between groups $\mathrm{D}$ and $\mathrm{C}$. TC levels were significantly lower in group $\mathrm{B}$ than groups $\mathrm{C}$ and $\mathrm{D}(\mathrm{P}<0.05$ and 0.01 , respectively). They were also significantly lower in group $\mathrm{DB}$ than group $\mathrm{D}(\mathrm{P}<0.05)$. The $\mathrm{LDL}$ levels were significantly lower in group DB than in group $\mathrm{D}(\mathrm{P}<0.05)$ (Table 1). Lastly, the testosterone levels were significantly lower in group $\mathrm{D}$ than groups $\mathrm{C}, \mathrm{B}$, and $\mathrm{DB}(\mathrm{P}<0.001$, 0.05 and 0.01 , respectively) (Table 1 ; Fig. 1 ).

\section{TAS, TOS, and OSI results}

The TAS levels were significantly higher in group B than group $\mathrm{C}(\mathrm{P}<0.05)$, while the TOS levels were significantly higher in group $\mathrm{D}$ than groups $\mathrm{C}$ and $\mathrm{DB}(\mathrm{P}<0.05)$. The OSI levels were significantly higher in group $D$ than group $C(P<0.05)$. They were also significantly lower in groups $\mathrm{B}$ and $\mathrm{DB}$ than group D $(\mathrm{P}<0.05)$ (Table 1; Fig. 2).

\section{Histopathological staining}

Microscopic examination showed that the prostate tissue was normal in groups C (Fig. 3a) and B (Fig. 3b). In group $\mathrm{D}$, the glandular lumens were filled with cellular debris and leucocytic infiltrate (Fig. 3c). The glandular epithelium was also degenerated or thickened in some regions. Administration of $B$. chrysogonum improved the general tissue structure and the epithelium was better preserved and less debris was seen in the glandular lumen in group DB than in group D (Table 2; Fig. 3d).

\section{Discussion}

Inflammation is a generalized histopathologic finding of BPH (Fibbi et al. 2010; Schauer and Rowley 2011) and is associated with a higher risk of lower urinary tract symptoms (LUTS) (Kramer and Marberger 2006) and acute urinary retention in aging men (Nickel et al. 2008). Recent studies have shown an increased risk of BPH in patients proven to have prostate inflammation on biopsy examination (Crawford et al. 2006). In prostate tissue developing hyperplasia, inflammation can induce proliferation events and nuclear deterioration through cytokines and oxidative stress (Naber and Weidner 2000). The aggressive release of oxygen radicals from macrophages and neutrophils during acute or chronic inflammation may lead to compensatory cellular proliferation, which further exacerbates oxidative stress, and in such conditions, hyperplastic proliferation may occur in the prostate tissue (Vital et al. 2015). Tanik et al. (2014) used inflammatory parameters like the neutrophil-to-lymphocyte ratio to predict $\mathrm{BPH}$ progression and LUTS severity and found a positive correlation between the neutrophil count and prostate hyperplasia.

High-fat diets (HFD) have also been implicated in oxidative stress and inflammation in the prostate gland (Shankar et al. 2012) as they increase the levels of acutephase inflammatory markers (Kriketos et al. 2004) via cytokines released from neutrophils and macrophages in adipose tissue (Shankar et al. 2015). Using a HFDinduced metabolic syndrome animal model with hyperglycemia, dyslipidemia, and hypogonadism, Vignozzi et al. (2012) linked metabolic syndrome to prostate inflammation, and found that it was correctible with testosterone replacement. Like HFD, DM has also been linked to chronic inflammatory conditions, and it has 
Table 1 Laboratory data and levels of oxidative status in groups (mean \pm SE)

\begin{tabular}{|c|c|c|c|c|}
\hline & Control & Diabetes (D) & Bongardia (B) & $D+B$ \\
\hline Glucose (mg/dL) & $234.1 \pm 16.72$ & $584.4 \pm 71.6^{\mathrm{a}}$ & $195.4 \pm 39.36^{b}$ & $307.6 \pm 51.8^{c}$ \\
\hline T. cholesterol (mg/dL) & $61.91 \pm 7.63$ & $61.52 \pm 3.79$ & $42.95 \pm 3.04^{c, d}$ & $45.77 \pm 5.00^{\mathrm{e}}$ \\
\hline Triglycerids (mg/dL) & $59.97 \pm 11.00$ & $56.42 \pm 6.01$ & $49.11 \pm 5.68$ & $52.68 \pm 7.04$ \\
\hline $\mathrm{HDL}(\mathrm{mg} / \mathrm{dL})$ & $16.01 \pm 1.84$ & $15.74 \pm 1.46$ & $10.72 \pm 1.66$ & $13.55 \pm 1.15$ \\
\hline LDL (mg/dL) & $30.94 \pm 4.31$ & $34.43 \pm 3.48$ & $25.68 \pm 4.11$ & $22.88 \pm 2.45^{\mathrm{e}}$ \\
\hline TAS (mmol/L) & $0.50 \pm 0.03$ & $0.66 \pm 0.03$ & $0.79 \pm 0.08^{d}$ & $0.61 \pm 0.05$ \\
\hline TOS (mmol/L) & $7.63 \pm 1.11$ & $13.79 \pm 1.21^{d}$ & $9.44 \pm 1.02$ & $7.97 \pm 1.85^{\mathrm{e}}$ \\
\hline OSI (=TOS/TAS) & $10.47 \pm 1.18$ & $19.15 \pm 1.97^{d}$ & $12.00 \pm 1.76^{e}$ & $12.94 \pm 0.90^{\mathrm{e}}$ \\
\hline Testosterone (mg/dL) & $2.78 \pm 0.48$ & $0.36 \pm 0.05^{\mathrm{a}}$ & $1.76 \pm 0.27^{e}$ & $0.94 \pm 0.14^{f, c}$ \\
\hline
\end{tabular}

$H D L$ high-density lipoprotein, LDL low-density lipoprotein, TAS total antioxidant status, TOS total oxidative status, OSI oxidative stress index

a $\mathrm{p}<0.001$

${ }^{b} \mathrm{p}<0.001$

${ }^{c} p<0.01$

${ }^{d} p<0.05$

e $p<0.05$ vs. diabetes

${ }^{f} \mathrm{p}<0.01$ vs. control

Table 2 The histopathology scores of prostate tissues (mean $\pm \mathrm{SE})$

\begin{tabular}{|c|c|c|c|c|}
\hline & Control & Diabetes (D) & Bongardia (B) & $D+B$ \\
\hline $\begin{array}{l}\text { Leucocytic } \\
\text { infiltrate }\end{array}$ & $0.20 \pm 0.13$ & $2.40 \pm 0.5^{\mathrm{a}}$ & $0.20 \pm 0.12^{c}$ & $1.30 \pm 0.15^{b, d}$ \\
\hline $\begin{array}{c}\text { Epithelial } \\
\text { degen- } \\
\text { eration }\end{array}$ & $0.10 \pm 0.10$ & $2.50 \pm 0.16^{a}$ & $0.20 \pm 0.13^{c}$ & $1.12 \pm 0.13^{b, d}$ \\
\hline \multicolumn{5}{|l|}{$\mathrm{a} p<0.0001$} \\
\hline \multicolumn{5}{|c|}{ b $\mathrm{p}<0.01$, vs. control } \\
\hline \multicolumn{5}{|c|}{${ }^{c} p<0.0001$} \\
\hline \multicolumn{5}{|c|}{${ }^{d} p<0.01$, vs. diabetes } \\
\hline
\end{tabular}

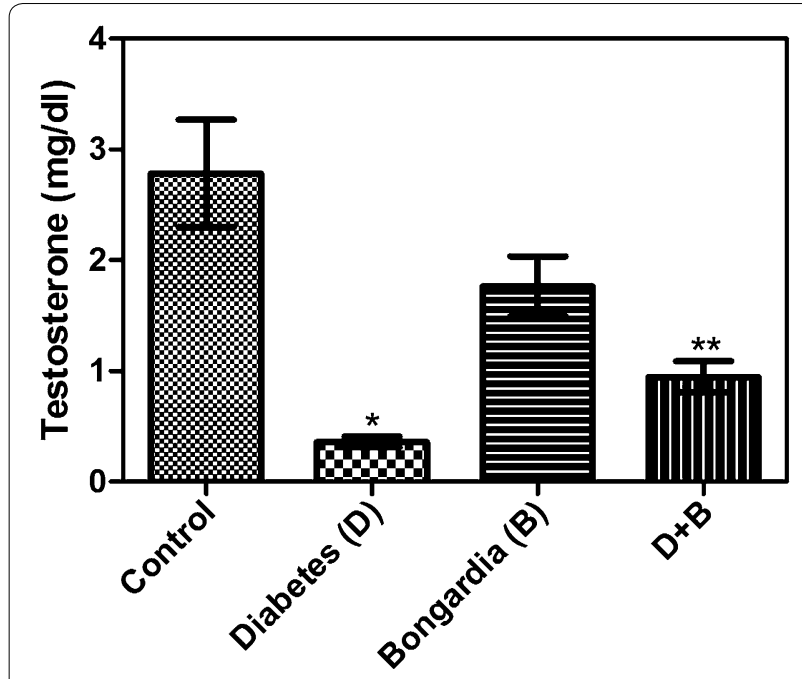

Fig. 1 Comparison of the testosterone levels in groups

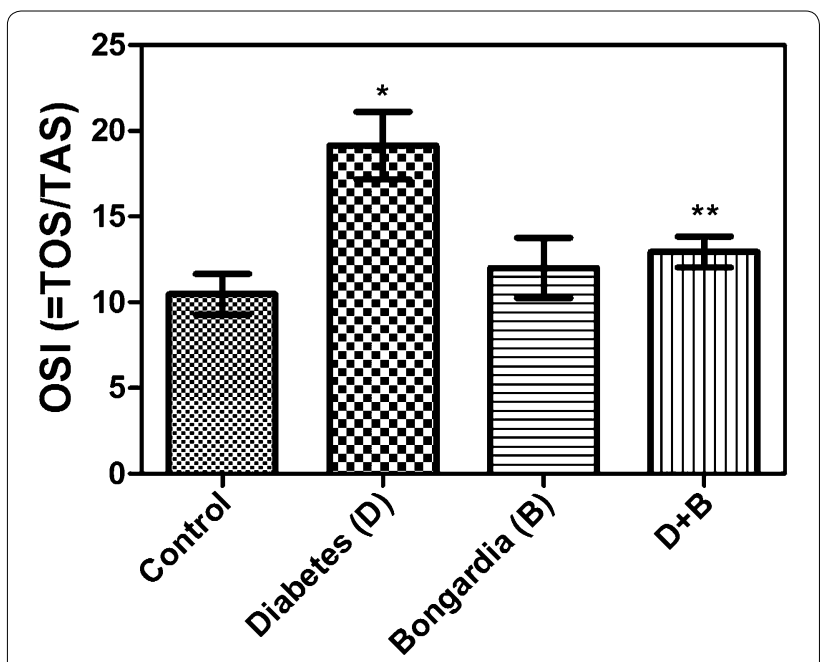

Fig. 2 Comparison of the oxidative stress index in groups

been implicated in increasing cytokine levels, mostly in the adipose tissue (Gibb and Strachan 2014). Further, DM-related androgen deficiency has been observed in a third of the population with type 2 diabetes, although the underlying mechanism remains unclear (Cheung et al. 2015; Morton 2005). The prostate volume has been found to be especially high in male patients diagnosed with type 2 diabetes (Kasturi et al. 2006), and in the NHANES-3 cohort study, Rohrman et al. observed a higher incidence of $\mathrm{BPH}$ in men with diabetes than in those without diabetes (American Diabetes Association 1998). Our results confirmed the effects of DM on prostate tissue shown in previous literature. A continuous supply of androgen 
is crucial for the growth and normal functioning of the prostate gland, and when this supply stops or decreases, prostate disease may develop (Yadav and Heemers 2012).

Rahman et al. (2000) conducted a detailed chemical examination of $B$. chrysogonum and examined the effects of saponins extracted from these tubers. Saponins are fat- and water-soluble glycosides with glycan or aglycan structures. In the intestine, saponins form micelles with bile acids, vitamins, fat acids, and diglycerides. Several studies have shown that because of a reduction in surface tension and detergent effects in the gastrointestinal tract, saponins reduce the emulsification of fat molecules and bile acids (Rao and Kendall 1986; Sidhu and Oakenfull 1986; Whitehead et al. 1981). Further, Bingham et al. (1978) showed that although saponins decreased the serum cholesterol and liver lipid concentrations, the liver cholesterol and serum HDL levels remain unchanged. The findings of our study agree with those of previous studies, as we clearly observed the anti-cholesterolemic and anti-lipidemic effects of B. chrysogonum in the blood and liver, respectively. Further, the testosterone levels in group D of the present study were significantly lower than those in group DB. These findings indicate that the hypogonadal status induced by DM was ameliorated by administration of $B$. chrysogonum. Additionally, correction of the deficiency in testosterone levels, which exacerbates prostate inflammation and tissue remodeling, may prevent or at least decelerate the course of $\mathrm{BPH}$.

Arslan et al. (2005) and Assaf et al. (2013) studied the effects (Fig. 3) of B. chrysogonum on various tissues under certain conditions. However, to our knowledge, ours is the first study to histologically examine its effects on prostate tissues in DM.

Histologically at the cellular level, BPH is characterized by basal cell hyperplasia, increased stromal mass, enhanced extracellular matrix deposition, reduced elastic tissue, increased infiltration of leucocytes around ducts, and acinar hypertrophy (Bostwick et al. 1992).

The accumulation of inflammation throughout the prostatic tissue aggravates the process of prostatic
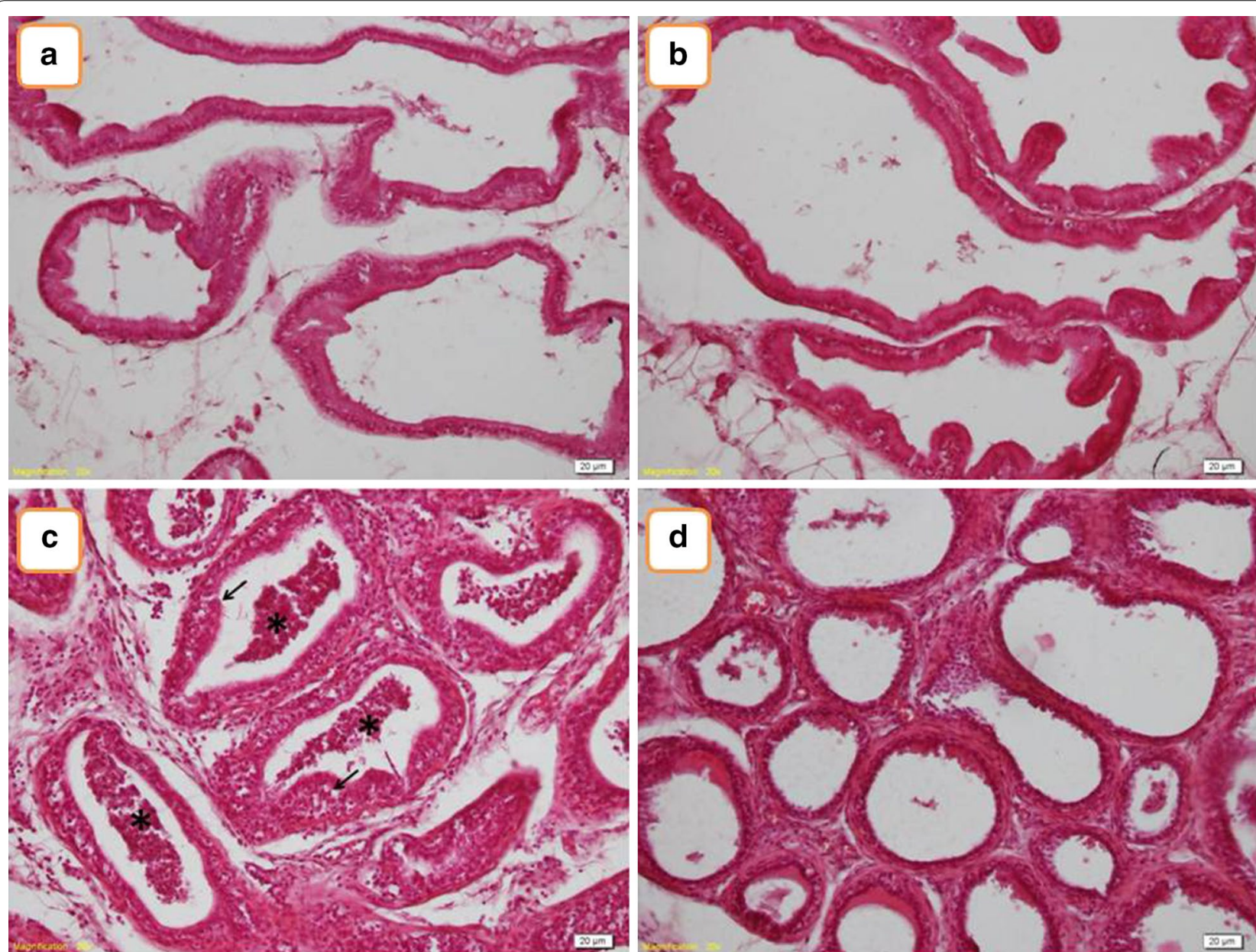

Fig. 3 Comparison of the histopathologic parameters in groups. a Control group; $\mathbf{b}$ diabet group; $\mathbf{c}$ bongardia group; d diabet+bongardia group 
hyperplasia by enhancing the production of prostatic growth factors. Furthermore, the altered secretion of luminal cells and clogging of the ducts with debris and corpora amylacea may lead to prostatic calcification, which is a significant modification of the hyperplastic prostate. Our findings showed that the conditions of the prostate tissue were better in rats with diabetes who were treated with $B$. chrysogonum than in those that were not treated with this herb: as mentioned in the "Results" section, the epithelium was better preserved and less debris was seen in the glandular lumen in group DB than in group D. Further, the TOS levels were significantly higher in group D than group DB, while the OSI levels were significantly lower.

Chronic conditions such as DM and hypercholesterolemia require lifelong treatment, and patient compliance may be better with alternative herbal treatment options in the case of such conditions. As indicated by our findings, the well-known plant $B$. chrysogonum seems to have positive effects on glucose and lipid metabolism. Further, it seems to prevent the pathological changes that occur in the prostate tissue in DM. Thus, this herb may be a useful treatment option for patients with DM and BPH. Further molecular studies are needed to confirm the effects of $B$. chrysogonum on the prostate tissue and to develop this herb into an effective therapeutic agent against DM and BPH.

\section{Abbreviations \\ DM: diabetes mellitus; BPH: benign prostate hyperplasia; TOS: total oxida- tive status; TAS: total antioxidant status; OSI: oxidative stress index; TC: total cholesterol; TG: triglycerides; LDL: low-density lipoprotein; HDL: high-density lipoprotein; HFD: high-fat diet; STZ: streptozotocin; LUTS: lower urinary tract symptoms.}

\section{Authors' contributions}

$\mathrm{RD}$ and $\mathrm{Ml}$ participated actively in the design of the experiment and functioned as mentors. KHG supervised the experiment and data analysis. OO, NKS, and AN performed histopathologic and biochemical data analysis and drafted the article. All authors read and approved the final manuscript.

\footnotetext{
Author details

1 Department of Medical Physiology, School of Medicine, Mustafa Kemal University, 31100 Antakya, Hatay, Turkey. ${ }^{2}$ Department of Urology, School of Medicine, Mustafa Kemal University, Hatay, Turkey. ${ }^{3}$ Department of Biochemistry, School of Medicine, Mustafa Kemal University, Hatay, Turkey. ${ }^{4}$ Department of Histology, School of Medicine, Mustafa Kemal University, Hatay, Turkey.

${ }^{5}$ Department of Histology, School of Medicine, Hacettepe University, Ankara, Turkey. ${ }^{6}$ Department of Information Technology, Lumina-The University of South-East Europe, Bucharest, Romania.

\section{Competing interests}

The authors declare that they have no competing interests.

\section{Ethical approval}

All applicable international, national, and/or institutional guidelines for the care and use of animals were followed. The ethics committee of Mustafa Kemal University approved the animal use protocol for this study in compliance with the guidelines of the Declaration of Helsinki (ID: 40595970/126).
}

\section{Funding}

This study was funded by the Mustafa Kemal University Scientific Research Projects Coordination Unit. (Number: 12457).

Received: 20 December 2015 Accepted: 29 July 2016

Published online: 11 August 2016

\section{References}

American Diabetes Association (1998) Screening for type II diabetes. Diabetes Care 21:520-522

Arslan ACE, Ozaslan M, Cengiz B, Bagci C, Tarakcioglu M, Sari I, Cekmen M, Karadag E, Kocabas R (2005) The effects of Bongardia chrysogonum, spach extract on the serum parameters and liver, kidney, and spleen tissues inrats. Biotechnol Biotechnol Eq 3:19

Assaf AM et al (2013) Anti-cancer, anti-inflammatory and anti-microbial activities of plant extracts used against hematological tumors in traditional medicine of Jordan. J Ethnopharmacol 145(3):728-736

Bingham R, Harris D, Laga T (1978) Yucca plant saponin in the treatment of hypertension and hypercholesterolemia. J Appl Nutr

Bostwick DG, Cooner WH, Denis L, Jones GW, Scardino PT, Murphy GP (1992) The association of benign prostatic hyperplasia and cancer of the prostate. Cancer 70(1 Suppl): 291-301

COMMITTEE AP (2003) AUA guideline on management of benign prostatic hyperplasia chapter 1: diagnosis and treatment recommendations. J Urol 170(2 Pt 1):530-547

Cheung KK et al (2015) Testosterone level in men with type 2 diabetes mellitus and related metabolic effects: a review of current evidence. J Diabetes Investig 6(2):112-123

Crawford ED et al (2006) Baseline factors as predictors of clinical progression of benign prostatic hyperplasia in men treated with placebo. J Urol 175(4):1422-1426 (Discussion 1426-7)

Dokuyucu R et al (2014) Antioxidant effect of erdosteine and lipoic acid in ovarian ischemia-reperfusion injury. Eur J Obstet Gynecol Reprod Biol 183:23-27

Erel O (2004) A novel automated direct measurement method for total antioxidant capacity using a new generation, more stable ABTS radical cation. Clin Biochem 37(4):277-285

Erel O (2005) A new automated colorimetric method for measuring total oxidant status. Clin Biochem 38(12):1103-1111

Fibbi B et al (2010) Chronic inflammation in the pathogenesis of benign prostatic hyperplasia. Int J Androl 33(3):475-488

Gibb FW, Strachan MW (2014) Androgen deficiency and type 2 diabetes mellitus. Clin Biochem 47(10-11):940-949

Gulturk S, Demirkazik A, Kosar I, Cetin A, Dökmetas H, Demir T (2010) Effect of exposure to $50 \mathrm{~Hz}$ magnetic field with or without insulin on blood brain barrier permeability in streptozotocin-induced diabetic rats. Bioelectromagnetics 31:262-269

Kasturi S, Russell S, McVary KT (2006) Metabolic syndrome and lower urinary tract symptoms secondary to benign prostatic hyperplasia. Curr Urol Rep 7(4):288-292

Klein R, Klein BE, Moss SE (2005) Ten-year incidence of self-reported erectile dysfunction in people with long-term type 1 diabetes. J Diabetes Complications 19(1):35-41

Kramer G, Marberger M (2006) Could inflammation be a key component in the progression of benign prostatic hyperplasia? Curr Opin Urol 16(1):25-29

Kriketos AD et al (2004) Inflammation, insulin resistance, and adiposity: a study of first-degree relatives of type 2 diabetic subjects. Diabetes Care 27(8):2033-2040

McVary K (2006a) Lower urinary tract symptoms and sexual dysfunction: epidemiology and pathophysiology. BJU Int 97(Suppl):223-228

McVary KT (2006b) BPH: epidemiology and comorbidities. Am J Manag Care 12(5 Suppl):S122-S128

Morton A (2005) Frequent occurrence of hypogonadotropic hypogonadism in type 2 diabetes. J Clin Endocrinol Metab 90(3):1903

Naber KG, Weidner W (2000) Chronic prostatitis-an infectious disease? J Antimicrob Chemother 46(2):157-161 
Nickel JC et al (2008) The relationship between prostate inflammation and lower urinary tract symptoms: examination of baseline data from the REDUCE trial. Eur Urol 54(6):1379-1384

Parsons JK (2007) Modifiable risk factors for benign prostatic hyperplasia and lower urinary tract symptoms: new approaches to old problems. J Urol 178(2):395-401

Rahman A et al (2000) Triterpenoid saponins from Bongardia chrysogonum. J Nat Prod 63(2):251-253

Rao AV, Kendall CW (1986) Dietary saponins and serum lipids. Food Chem Toxicol 24(5):441

Rosen $R$ et al (2003) Lower urinary tract symptoms and male sexual dysfunction: the multinational survey of the aging male (MSAM-7). Eur Urol 44(6):637-649

Rosen RC, Giuliano F, Carson CC (2005) Sexual dysfunction and lower urinary tract symptoms (LUTS) associated with benign prostatic hyperplasia (BPH). Eur Urol 47(6):824-837

Schauer IG, Rowley DR (2011) The functional role of reactive stroma in benign prostatic hyperplasia. Differentiation 82(4-5):200-210

Shankar E et al (2012) High-fat diet activates pro-inflammatory response in the prostate through association of Stat-3 and NF-kappaB. Prostate 72(3):233-243
Shankar E et al (2015) Inflammatory signaling involved in high-fat diet induced prostate diseases. J Urol Res 2(1)

Sidhu GS, Oakenfull DG (1986) A mechanism for the hypocholesterolaemic activity of saponins. Br J Nutr 55(3):643-649

Tanik $S$ et al (2014) Is the neutrophil-lymphocyte ratio an indicator of progression in patients with benign prostatic hyperplasia? Asian Pac J Cancer Prev 15(15):6375-6379

Vignozzi L et al (2012) Testosterone protects from metabolic syndrome-associated prostate inflammation: an experimental study in rabbit. J Endocrinol 212(1):71-84

Vital P. Castro P. Ittmann M (2015) Oxidative stress promotes benign prostatic hyperplasia. Prostate 76:58-67

Whitehead CC, McNab JM, Griffin HD (1981) The effects of low dietary concentrations of saponin on liver lipid accumulation and performance in laying hens. Br Poult Sci 22(3):281-288

Yadav N, Heemers HV (2012) Androgen action in the prostate gland. Minerva Urol Nefrol 64(1):35-49

\section{Submit your manuscript to a SpringerOpen ${ }^{\circ}$ journal and benefit from:}

- Convenient online submission

- Rigorous peer review

- Immediate publication on acceptance

- Open access: articles freely available online

- High visibility within the field

- Retaining the copyright to your article 